\title{
EFFECTS OF SHADE AND WATER STRESS CN GROWTH AND RELATED PHYSIOLOGICAL PARAMETERS OF THE SEEDLINGS OF FIVE FOREST TREE SPECIES
}

\author{
W.A.J.M. DE COSTA* and M.F. ROZANA \\ Department of Crop Science, Faculty of Agriculture, University of Peradeniya, Peradeniya.
}

(Received: 02 March 1999 ; accepted: 11 February 2000)

\begin{abstract}
The main objective of the present study was to investigate the effects of different levels of shade and water: availability on tree seedling growth and on some related physiological parameters. The five tree species were selected to represent two early successional species (i.e. Alstonia macrophylla and Acacia auriculiformis), two late-successional species (i.e. Artocarpus heterophyll.us and Terminalia arjun.a) and one dry zone species (i.e. Azadiracta indica). Even-aged seedlings of the tree species were grown in pots in a plant house under three levels of artificial shade,i.e. open, medium ( $40 \%$ shade) and high (70\%) shade, and two water regimes, i.e. well-watered and water-stressed up to temporary early-morning wilting, during the period from 27 May to 10 November; 1998.
\end{abstract}

Both absolute and relative biomass gain of tree species during the experimental period decreased significantly with increasing shade. Alstonia had the highest relative growth rates under all shade $\mathrm{x}$ water regime combinations whereas Azudiractu had the lowest. Significant inter-pocios variation was shown in leaf water potential ( $\Psi$ ), leaf stomatal conductance ( $g_{1}$ ), leaf chlorophyll (LCC), nitrogen (LNC) and potassium (LPC) contents under all shade $\mathrm{x}$ water regime combinations. Water stress decreased biomass gain, LCC and $g_{1}$ and increased LNC. Increasing shade decreased $g_{1}$ and leaf weight ratio, but increased root weight ratio under both water regimes. However, there were significant shade $\mathrm{x}$ water regime interaction effects on LCC, LNC and $\Psi$. Both absolute biomass gain and relative growth rate ( $R G R$ ) had significant positive correlations with $\Psi^{\prime}, g_{1}$ and LCC but were not correlated with LNC or LPC. Multiple regression analysis identified $\Psi$ and $g_{1}$ as the factors which contributed most to the observed variation of absolute biomass gain and RGR.

Key words: Acacia auriculiformis, Alstonia macrophyll.a, Artocarpus heterophyllus, Azadiracta ind.icu, forest trees, relative growth rate, shade, Terminalia arjuna, water stress

\section{INTRODUCTION}

Significant deforestation has reduced the closed-canopy forest cover in Sri Lanka from around $80 \%$ in 1880 to $22 \%$ in 1995 (Forest department, personal communication). Although most deforestation in recent times has occurred in the dry and intermediate zones, even a smaller degree of deforestation in the wet zone can have greater ecological and environmental impacts. ${ }^{1}$ Therefore, there is an

${ }^{*}$ Corresponding author 
urgent need to increase the existing forest cover through reforestation and supporting natural regeneration. Proper selection of tree species is important for this process. Hence, the present paper evaluates the growth and its physiological basis of some selected tree species at seedling stage.

Seedling survival and growth within a forest is determined to a large extent by the environmental conditions. ${ }^{2}$ The degree of shade created by the canopy is a key parameter as it determines the amount of radiant energy available for photosynthesis in growing seedlings. ${ }^{.}$In general, pioneer and early-successional species require high light levels to maintain a positive carbon balance in their seedlings while the late-successional and climax species are able to survive more shaded conditions. ${ }^{4}$ Even for these climax species, high light intensities provided through occasional sunflecks play an important role in maintaining a positive carbon balance..$^{5, i}$

Soil water also is a key parameter in seedling survival and growth because of the sensitivity of photosynthesis to water availability. Water stress inhibits photosynthesis through both stomatal and non-siomatal (i.e. biochemical) effects. ${ }^{7}$ Stomatal effects of water stress occur due to the reduction of stomatal conductance (i.e. partial closure of stomata) and the consequent reduction in the influx of $\mathrm{CO}_{2}$ into the leaves which decreases the photosynthetic rate. ${ }^{\circ}$ On the other hand, non-stomatal effects of water stress involve substantial reduction of reaction rates in different steps of the Calvin cycle." Moreover, a low water balance within the plant causes a reduction in cellular turgor which inhibits several other physiological processes responsible for plant growth such as synthesis of cell wall material and cellular expansion. ${ }^{10}$

In conditions found within natural forests, there is likely to be an interaction between the effects of shade and water availability on the survival and growth of different forest tree species. For example, it may be possible for a given tree species to tolerate a higher degree of water stress under shaded conditions because of lower transpiration rates. On the other hand, growth can be accelerated when open conditions coincide with greater water availability. In addition, the inherent difference between early-and late-successional species in their preference of open or shaded conditions,,$" 4$ introduces a further factor which complicates the understanding of the physiological basis of tree growth in a natural forest. Disentagling the individual effects of the above complicating factors require measurements under controlled conditions as specific shade and water treatments cannot be imposed under natural forest-growing conditions.

The present study was done to compare the effects of different levels of shade and water availability on biomass production and on selected physiological and morphological parameters in five tree species representing both early-and late-successional groups and wet zone/ dry zone species groups. 


\section{METHODS AND MATERIALS}

Experimental location : The experiment was conducted at the rain-sheltered plant house at the Faculty of Agriculture of the University of Peradeniya from May to November, 1999. The experimental site is located at an elevation of $479.9 \mathrm{~m}$ in the mid-country wet zone. ${ }^{11}$ The ambient mean temperature was $26-28^{\prime \prime} \mathrm{C}$ and the mean relative humidity was $75-80 \%$.

Planting material and plant establishment: Even-aged, uniform seedlings of five tree species (Acacia auriculiformis, Alstonia macrophylla, Artocarpus heterophyllus, Terminalia arjuna and Azadiracta indica) collected from nurseries were used. Among these, Acacia and Alstonia are considered as early-successional species whereas Artocarpus and Terminalia are considered as late-successional species. Azadiracta is a species specially adapted to grow in the dry zone whereas the others are more suited to the relatively more moist conditions found in the wet zone. Seedlings were established in plastic pots which were of $20 \mathrm{~cm}$ diameter and $15 \mathrm{~kg}$ capacity on 23 May 1998. The soil used was a mixture of top and sub-soil obtained from the experimental site and belonged to the great group Red Yellow Podzolic. ${ }^{11}$

Experimental treatments: The treatment structure was a three-factor factorial arranged in a strip-plot design. ${ }^{12}$ The main plot consisted of three levels of shade imposed by green, plastic shade netting. The three shade levels were termed 'open', 'medium-shade' and 'high-shade'. The open treatment was covered only by the transparent polythene roof of the rainshelter and received $90 \%$ of incident radiation. The medium shade treatment was created with one layer of shade netting and received $60 \%$ of incident radiation. The high shade treatment had two layers of shade netting and received $30 \%$ of incoming radiation. Within each shading level, the five tree species and two water levels were completely randomized. One of the two water levels represented a 'well-watered' treatment which was irrigated daily and was maintained with a non-limiting supply of water. The 'water-stressed' treatment underwent repeated drying cycles during the experimental period. During a drying cycle, water was withheld until a plant showed temporary wilting symptoms early in the morning. With the onset of wilting symptoms, the respective plants were watered up to $50 \%$ of available water (i.e. the difference between pre-determined soil water contents at field capacity and permanent wilting point). There were two replicates of main plots and within each main plot there were six replicates for each species $\mathrm{x}$ water level combination. The treatments were imposed on 27 May 1998.

Plants were maintained free of pests and diseases. No organic or inorganic fertilizers were added during the experimental period. 
Measurements: Initial measurements: At the beginning of the experiment, dry weights of leaves, stems and roots were measured by destructive sampling of two plants per treatment combination. Dry weights were obtained by oven-drying the respective plant parts at $80^{\circ} \mathrm{C}$ to a constant weight. Dried leaf samples were then ground and used for nutrient analysis.

Leaf stomatal conductance $\left(\mathrm{g}_{\mathrm{g}}\right)$ : Leaf stomatal conductance $\left(\mathrm{g}_{\mathrm{g}}\right)$ was measured on three days (27 August, 03 September and 29 September 1998) during the experimental period using an automatic diffusion porometer (Delta-T Devices, UK). At these times, the water-stress treatments of all plant species were at an equivalent stage in the middle of their drying cycles. The porometer was calibrated at the beginning of each day of measurement. Measurements were done between 1200 and 1400 hours when the effects of water stress on $g_{1}$ was expected to be greatest. Both the upper- and lower-surface conductances were measured and the total leaf conductance was obtained by summing the conductances of the two sides. The first fully-expanded leaf was used for measurements. Four replicate measurements were taken in each treatment combination.

Leaf' water potential ( $\Psi$ ): Water potential of fully-expanded, young leaves were measured using the pressure chamber. ${ }^{13}$ LWP measurements were carried out on three days (28 August, 04 September and 30 September 1998) on the days immediately following the three stomatal conductance measurements. Two replicate measurements were done in each treatment combination. Measurements were made immediately after excision of leaves while taking precautions to minimize possible errors. ${ }^{1.4}$

Leaf chlorophyll and nutrient contents: Leaf chlorophyll was extracted by acetone $(80 \% \mathrm{v} / \mathrm{v})$ and absorbance of the extracted solution was measured by the spectrophotometer at $645 \mathrm{~nm}$ (for chlorophyll a) and $663 \mathrm{~nm}$ (for chlorophyll b) wavelengths. ${ }^{15}$ Total chlorophyll content was computed by adding the contents of chlorophyll $\mathrm{a}$ and $\mathrm{b}$.

Final measurements: Plants were destructed for final biomass measurements on 10 November 1998. Dry weights of leaves, stems and roots were measured as described before. Biomass gain during the 167-day experimental period was computed as the difference between the final and the initial biomass values. Relative growth rate (RGR) during the experimental period was computed as:

$$
R G R=\frac{\left(\log _{\mathrm{e}} \mathrm{W}_{\mathrm{f}}-\log _{\mathrm{e}} \mathrm{W}_{\mathrm{i}}\right)}{\mathrm{R}}
$$


where, $W_{\text {, and }} W_{i}$ are the final and the initial total dry weights and $t$ is the experimental duration (i.e. 167 days). Gain in leaf weight ratio (LWR) was calculated as the ratio between leaf dry weight increase and total dry weight increase ${ }^{16}$ during the experimental period. Similarly, increase in root weight ratio (RWR) was computed as the ratio between the gains in root and total dry weights.

Leaf nitrogen content was measured by the micro-Kjeldahl method. ${ }^{17}$ Leaf potassium content was measured by the dry ash method using the flame photometer. ${ }^{\text {is }}$

Data analysis: Significance of treatment differences were tested by analysis of variance and mean separation was done by using the least significant difference. ${ }^{1 !}$ Strengths of the relationships between biomass gain and the measured leaf characters were analyzed by multiple correlation analysis. ${ }^{12}$ Contribution of each of the leaf characters to the observed variation in biomass gain was estimated by multiple regression analysis ${ }^{1 ! 2}$ using the stepwise rerression procedure. The full regression models used were,

$$
\begin{aligned}
& W=a+b \Psi+c g_{1}+d(L C C)+e(L N C)+f(L P C)+\text { error } \\
& R G K=a+b \Psi+c g_{1}+d(L C C)+e(L N C)+f(L P C)+\text { error }
\end{aligned}
$$

where $W$ and $R G R$ were absolute biomass gain and relative growth rate respectively and $b, c, d$, e and $f$ were the respective regression coefticients. The contribution of each variable to the observed variation of $W$ or $R G R$ was estimated by its partial $R^{2}$ value (i.e. the percentage of total sums of squares explained by the variable being considered).

\section{RESULTS}

\section{Total biomass gain}

Analysis of variance (ANOVA) showed that all three factors examined (i.e. species, shade and water) had highly significant $(p<0.01)$ effects on total biomass gain during the experimental period. Table 1 shows the growth of different tree species under different shade and water regimes. When averaged across different shade levels, Terminalia and Alstoniu showed significantly greater growth than the rest under both well-watered (WW) and water-stressed (WS) conditions. In contrast, under both water regimes, Acacia and Azadiracta had the lowest biomass gain while Artocarpus had intermediate levels. Within well-watered conditions, growth under open conditions was significantly greater $(p<0.05)$ than that at medium or high shade which did not differ significantly (Table 1). Under water stress, while maintaining the superior growth in open conditions, a significantly greater biomass gain was shown under medium shade as compared to 
high shade. Water stress reduced the biomass gain of all species at all shade levels and the overall growth under water-stressed conditions was significantly $(p<0.05)$ lower than under well-watered conditions.

Table 1: Increase in total biomass (g/plant) of different tree species under varying shade and water regimes

\begin{tabular}{|c|c|c|c|c|c|c|c|c|}
\hline \multirow{2}{*}{ Tree species } & \multicolumn{5}{|c|}{ Well - watered } & \multicolumn{3}{|c|}{ Water - stressed } \\
\hline & Open & Med. & High & Mean & Open & Med. & High & Mean $^{*}$ \\
\hline $\begin{array}{l}\text { Alstonica } \\
\text { rnacrophylla }\end{array}$ & 21.94 & 10.94 & 10.27 & $\begin{array}{c}14.38 \\
\mathrm{~A}\end{array}$ & 17.26 & 9.90 & 6.84 & $\begin{array}{c}11.33 \\
\mathrm{~A}\end{array}$ \\
\hline $\begin{array}{l}\text { Artocarpus } \\
\text { heterophyllus }\end{array}$ & 6.21 & 5.02 & 4.37 & $\begin{array}{c}5.20 \\
\mathrm{~B}\end{array}$ & 6.90 & 2.60 & 3.08 & $\begin{array}{c}4.19 \\
\mathrm{C}\end{array}$ \\
\hline $\begin{array}{l}\text { Acacia } \\
\text { auriculiformis }\end{array}$ & 3.13 & 1.00 & 0.95 & $\begin{array}{c}1.69 \\
\mathrm{C}\end{array}$ & 2.09 & 1.12 & 0.88 & $\begin{array}{c}1.36 \\
\mathrm{E}\end{array}$ \\
\hline $\begin{array}{l}\text { Azadiracter } \\
\text { indica }\end{array}$ & 5.70 & 1.78 & 2.24 & $\begin{array}{c}3.24 \\
\mathrm{C}\end{array}$ & 2.93 & 3.33 & 1.90 & $\begin{array}{c}2.72 \\
D\end{array}$ \\
\hline $\begin{array}{l}\text { Terminalia } \\
\text { arjuna. }\end{array}$ & 21.94 & 12.16 & 10.22 & $\begin{array}{c}14.77 \\
\mathrm{~A}\end{array}$ & 12.99 & 9.29 & 5.84 & $\begin{array}{c}9.37 \\
\mathrm{~B}\end{array}$ \\
\hline $\begin{array}{l}\text { Meant } \\
\text { (Shade) }\end{array}$ & $\begin{array}{c}11.78 \\
\mathrm{a}\end{array}$ & $\begin{array}{c}6.18 \\
b\end{array}$ & $\begin{array}{c}5.61 \\
\mathrm{~b}\end{array}$ & & $\begin{array}{c}8.43 \\
\mathrm{a}\end{array}$ & $\begin{array}{c}5.25 \\
b\end{array}$ & $\begin{array}{c}3.71 \\
\mathrm{c}\end{array}$ & \\
\hline Mean (Water) & & & $7.86 \mathrm{a}$ & & & & $5.80 \mathrm{~b}$ & \\
\hline
\end{tabular}

Mean value for each species within each water level

"Mean value for each shade leve] within each wate? level

Means with the same letter are not significantly difterent at $p=0.05$

Absolute gains in leaf and root dry weights of the different tree species in response to shade and water regimes (data not shown), showed a variation pattern which was similar to that of total biomass gain. Tables 2 and 3 show the variations in leaf weight ratio (LWR) and root weight ratio (RWR) under different shade and water levels. There were highly significant $(p<0.01)$ differences between species and shade regimes for both LWR and RWR. However, both these parameters were not affected significantly $(\mathrm{p}<0.05)$ by variation in water availability. Alstonia had the highest LWR among the tree species tested under both well-watered and water-stressed conditions (Table 2). On the other hand, Terminalia had lower LWR under both water regimes with Ázadiracta also having a very low LWR under water-stress. High shade reduced LWR significantly $(\mathrm{p}<0.05)$ under both water regimes whereas LWR did not differ significantly between open and medium shade 
(Table 2). Highest RWR was shown by Terminalia under both water regimes (Table 3). In contrast, the lowest RWR was observed in Artocarpus under well-watered conditions and in Alstonia under water stress. RWR was significantly lower under open conditions as compared to medium and high shade in both water regimes. There was significant species $x$ water regime interaction in both $L W R$ and RWR, with some species increasing these parameters and the other decreasing them in response to water stress (Table 3 ).

Table 2: Increase in leaf weight ratio $\left(\mathrm{g} \mathrm{g}^{-1}\right)$ of different tree species under varying shade and water regimes

\begin{tabular}{|c|c|c|c|c|c|c|c|c|}
\hline \multirow{2}{*}{ Tree species } & \multicolumn{4}{|c|}{ Well - watered } & \multicolumn{3}{|c|}{ Water - stressed } & \multirow[b]{2}{*}{ Mean* } \\
\hline & Open & Med. & High & Mean ${ }^{*}$ & Open & Med. & High & \\
\hline $\begin{array}{l}\text { Alstonia } \\
\text { macrophylla }\end{array}$ & 0.38 & 0.42 & 0.27 & $\begin{array}{c}0.36 \\
\mathrm{~A}\end{array}$ & 0.35 & 0.36 & 0.43 & $\begin{array}{c}0.38 \\
\mathrm{~A}\end{array}$ \\
\hline $\begin{array}{l}\text { Artocarpus } \\
\text { heterophyllus }\end{array}$ & 0.19 & 0.23 & 0.10 & $\begin{array}{c}0.17 \\
\mathrm{BC}\end{array}$ & 0.08 & 0.09 & 0.14 & $\begin{array}{c}0.10 \\
\mathrm{~B}\end{array}$ \\
\hline $\begin{array}{l}\text { Acacia } \\
\text { auriculiformis }\end{array}$ & 0.30 & 0.25 & 0.08 & $\begin{array}{c}0.21 \\
\mathrm{~B}\end{array}$ & 0.40 & 0.49 & 0.11 & $\begin{array}{c}0.33 \\
\mathrm{~A}\end{array}$ \\
\hline $\begin{array}{l}\text { Azadiracta } \\
\text { indica }\end{array}$ & 0.12 & 0.43 & 0.19 & $\begin{array}{c}0.25 \\
\mathrm{~B}\end{array}$ & 0.11 & 0.08 & 0.06 & $\begin{array}{c}0.09 \\
\mathrm{~B}\end{array}$ \\
\hline $\begin{array}{l}\text { Terminalia } \\
\text { arjuna }\end{array}$ & 0.19 & 0.07 & 0.12 & $\begin{array}{c}0.13 \\
\mathrm{C}\end{array}$ & 0.06 & 0.12 & 0.09 & $\begin{array}{c}0.09 \\
B\end{array}$ \\
\hline $\begin{array}{l}\text { Mean } \\
\text { (Shade) }\end{array}$ & $\begin{array}{c}0.24 \\
\mathrm{a}\end{array}$ & $\begin{array}{c}0.28 \\
\mathrm{a}\end{array}$ & $\begin{array}{c}0.15 \\
\mathrm{~b}\end{array}$ & & $\begin{array}{c}0.20 \\
\mathrm{ab}\end{array}$ & $\begin{array}{c}0.23 \\
\mathrm{a}\end{array}$ & $\begin{array}{c}0.17 \\
b\end{array}$ & \\
\hline Mean (water) & & & $0.22 \mathrm{a}$ & & & & $0.20 \mathrm{a}$ & \\
\hline
\end{tabular}

- Mean value for each species within each water level

Mean value for each shade level within each water leve]

Means with the same letter are not significantly different at $\mathrm{p}=0.05$

\section{Relative growth rates ( $\mathrm{RGR})$}

Because of the differences in initial seedling dry weights between different species, RGR was computed as the biomass gain per unit of existing total dry weight. Similar to the absolute gain in biomass, RGR also showed highly significant $(p<0.001)$ variation between species, shade and water regimes. Alstonia had significantly $(p<0.05)$ greater RGR under both water regimes (Table 4) while Artocarpus also 
Table 3: Increase in root weight ratio $\left(\mathrm{g} \mathrm{g}^{-1}\right)$ of different tree species under varying shade and water regimes

\begin{tabular}{|c|c|c|c|c|c|c|c|c|}
\hline \multirow{2}{*}{ Tree species } & \multicolumn{4}{|c|}{ Well - watered } & \multicolumn{3}{|c|}{ Water - stressed } & \multirow[b]{2}{*}{ Mean } \\
\hline & Open & Med. & High & Mean ${ }^{*}$ & Open & Med. & High & \\
\hline $\begin{array}{l}\text { Alstonia } \\
\text { macrophylla }\end{array}$ & 0.20 & 0.44 & 0.44 & $\begin{array}{l}0.36 \\
A B\end{array}$ & 0.22 & 0.34 & 0.24 & $\begin{array}{c}0.27 \\
\mathrm{C}\end{array}$ \\
\hline $\begin{array}{l}\text { Artocarpus } \\
\text { heterophyllus }\end{array}$ & 0.37 & 0.19 & 0.19 & $\begin{array}{c}0.25 \\
\mathrm{~B}\end{array}$ & 0.31 & 0.42 & 0.38 & $\begin{array}{l}0.37 \\
\mathrm{AB}\end{array}$ \\
\hline $\begin{array}{l}\text { Acacia } \\
\text { auriculiformis }\end{array}$ & 0.38 & 0.25 & 0.38 & $\begin{array}{l}0.34 \\
\mathrm{AB}\end{array}$ & 0.34 & 0.27 & 0.31 & $\begin{array}{l}0.30 \\
\mathrm{BC}\end{array}$ \\
\hline $\begin{array}{l}\text { Azadiracta } \\
\text { indica }\end{array}$ & 0.17 & 0.26 & 0.54 & $\begin{array}{l}0.32 \\
A B\end{array}$ & 0.18 & 0.49 & 0.17 & $\begin{array}{l}0.28 \\
\mathrm{BC}\end{array}$ \\
\hline $\begin{array}{l}\text { Terminalia } \\
\text { arjuna }\end{array}$ & 0.36 & 0.49 & 0.53 & $\begin{array}{c}0.46 \\
\mathrm{~A}\end{array}$ & 0.31 & 0.32 & 0.77 & $\begin{array}{c}0.47 \\
\mathrm{~A}\end{array}$ \\
\hline $\begin{array}{l}\text { Meant } \\
\text { (shade) }\end{array}$ & $\begin{array}{c}0.30 \\
b\end{array}$ & $\begin{array}{c}0.33 \\
\mathrm{ab}\end{array}$ & $\begin{array}{c}0.41 \\
\mathrm{a}\end{array}$ & & $\begin{array}{c}0.27 \\
b\end{array}$ & $\begin{array}{c}0.37 \\
\mathrm{a}\end{array}$ & $\begin{array}{c}0.38 \\
a\end{array}$ & \\
\hline Mean (water) & & & $0.34 \mathrm{a}$ & & & & $0.34 \mathrm{a}$ & \\
\hline
\end{tabular}

Mean value lor each species within each water level

+ Mean value for each shade level within each water level

Means with the sume letter are not significantly different at $p=0.05$

had higher RGR levels than the rest of the species. In contrast, Azadiracta and Terminalia had significantly $(\mathrm{p}<0.05)$ lower RGR under both water regimes. There was a significant decrease of RGR with increasing shade under both well-watered and water-stressed conditions. Water stress decreased RGR in all species under all shade levels.

\section{Leaf chlorophyll content (LCC)}

There were highly significant $(\mathrm{p}<0.01)$ species, shade and water regime effects on leaf chlorophyll content. Alstonia showed the highest LCC under well-watered conditions (Table 5) while Terminalia had the highest LCC under water stress. In contrast, Acacia had the lowest LCC under both water regimes with Azadiracta. also having a lower LCC under water stress (Table 5). When averaged across different species and shade levels, there was a significant decrease of LCC under water-stress relative to that under well-watered conditions. Because of the 
significant shade $x$ species interaction, LCC did not show a clear variation pattern with increasing shade.

Table 4: Relative growth rate $\left(\mathrm{mg} \mathrm{g}^{-1} \mathrm{~d}^{-1}\right)$ of different tree species under varying shade and water regimes

Well-watered Water-stressed

Tree species

\begin{tabular}{|c|c|c|c|c|c|c|c|c|}
\hline 然 & Open & Med. & High & Mean & Open & Med. & High & Mean' \\
\hline $\begin{array}{l}\text { Alstonia } \\
\text { macrophylla }\end{array}$ & 11.03 & 7.76 & 7.46 & $\begin{array}{c}8.75 \\
\mathrm{~A}\end{array}$ & 9.84 & 7.34 & 5.85 & $\begin{array}{c}7.68 \\
\mathrm{~A}\end{array}$ \\
\hline $\begin{array}{l}\text { Artocarpus } \\
\text { heterophyllus }\end{array}$ & 7.34 & 6.70 & 6.15 & $\begin{array}{c}6.73 \\
B\end{array}$ & 8.06 & 4.35 & 4.90 & $\begin{array}{c}5.77 \\
\mathrm{C}\end{array}$ \\
\hline $\begin{array}{l}\text { Acacia } \\
\text { auriculiformis }\end{array}$ & 5.59 & 2.55 & 2.43 & $\begin{array}{c}3.52 \\
\mathrm{C}\end{array}$ & 4.47 & 2.80 & 2.25 & $\begin{array}{c}3.17 \\
\mathrm{E}\end{array}$ \\
\hline $\begin{array}{l}\text { Azadiracta } \\
\text { indica }\end{array}$ & 3.92 & 1.51 & 1.85 & $\begin{array}{c}2.43 \\
\mathrm{CD}\end{array}$ & 2.32 & 2.58 & 1.60 & $\begin{array}{c}2.17 \\
D\end{array}$ \\
\hline $\begin{array}{l}\text { Terminalia } \\
\text { arjuna }\end{array}$ & 4.22 & 2.70 & 2.34 & $\begin{array}{c}3.09 \\
\mathrm{D}\end{array}$ & 2.85 & 2.16 & 1.40 & $\begin{array}{c}2.14 \\
B\end{array}$ \\
\hline $\begin{array}{l}\overline{\text { Meant }} \\
\text { (Shade) }\end{array}$ & $\begin{array}{c}6.42 \\
a\end{array}$ & $\begin{array}{c}4.25 \\
b\end{array}$ & $\begin{array}{c}4.05 \\
\mathrm{~b}\end{array}$ & & $\begin{array}{c}5.51 \\
\mathrm{a}\end{array}$ & $\begin{array}{c}3.84 \\
b\end{array}$ & $\begin{array}{c}3.20 \\
c\end{array}$ & \\
\hline Mean (Water) & & & $4.90 \mathrm{a}$ & & & & $4.18 \mathrm{~b}$ & \\
\hline
\end{tabular}

- Mean value for each species within each water level

* Mean value for each shade level within each water level

Means with the same letter are not significantly different at $p=0.05$

\section{Leaf nitrogen content (LNC)}

There were significant $(p<0.05)$ species and water regime effects on leaf nitrogen content. Although the main effect of shade regimes on LNC was not significant at $p=0.05$, effects of the two-way interactions shade $x$ species and shade $x$ water regimes were highly significant. Under well-watered conditions, there was an increasing trend of LNC with increasing shade (Table 6). However, the opposite trend was observed under water-stressed conditions. Azadiracta had the highest LNC under both water regimes (Table 6) whereas Acacia had lower LNC. When averaged across different species and shade regimes, the mean LNC under water stress was significantly greater $(p<0.05)$ than that under well-watered conditions. This increase in LNC in response to water stress was shown by all species (Table 6). 
Table 5: Leaf chlorophyll content $\left(\mathrm{mg} \mathrm{g}^{-1}\right.$ leaf fresh weight) of different tree species under varying shade and water regimes

\begin{tabular}{|c|c|c|c|c|c|c|c|c|}
\hline \multirow{2}{*}{ Tree species } & \multicolumn{4}{|c|}{ Well - watered } & \multicolumn{3}{|c|}{ Water - stressed } & \multirow[b]{2}{*}{ Mean } \\
\hline & Open & Med. & High & Mean & Open & Med. & High & \\
\hline $\begin{array}{l}\text { Alstonia } \\
\text { macrophylla }\end{array}$ & 0.52 & 0.40 & 0.51 & $\begin{array}{c}0.48 \\
\mathrm{~A}\end{array}$ & 0.28 & 0.44 & 0.45 & $\begin{array}{c}0.39 \\
\mathrm{~B}\end{array}$ \\
\hline $\begin{array}{l}\text { Artocarpus } \\
\text { herterophyllus }\end{array}$ & 0.44 & 0.39 & 0.39 & $\begin{array}{c}0.41 \\
\mathrm{~A}\end{array}$ & 0.35 & 0.38 & 0.34 & $\begin{array}{c}0.36 \\
\mathrm{~B}\end{array}$ \\
\hline $\begin{array}{l}\text { Acacia } \\
\text { auriculiformis }\end{array}$ & 0.33 & 0.24 & 0.28 & $\begin{array}{c}0.28 \\
\mathrm{D}\end{array}$ & 0.28 & 0.32 & 0.27 & $\begin{array}{l}0.29 \\
\mathrm{C}\end{array}$ \\
\hline $\begin{array}{l}\text { Azadiracta } \\
\text { indica }\end{array}$ & 0.40 & 0.32 & 0.34 & $\begin{array}{c}0.35 \\
\mathrm{C}\end{array}$ & 0.28 & 0.32 & 0.28 & $\begin{array}{c}0.29 \\
\mathrm{C}\end{array}$ \\
\hline $\begin{array}{l}\text { Terminalia } \\
\text { arjuna }\end{array}$ & 0.41 & 0.27 & 0.52 & $\begin{array}{l}0.40 \\
\mathrm{BC}\end{array}$ & 0.54 & 0.38 & 0.43 & $\begin{array}{c}0.44 \\
\mathrm{~A}\end{array}$ \\
\hline $\begin{array}{l}\text { Mean }{ }^{\dagger} \\
\text { (shade) }\end{array}$ & $\begin{array}{c}0.42 \\
\mathrm{a}\end{array}$ & $\begin{array}{c}0.32 \\
b\end{array}$ & $\begin{array}{c}0.40 \\
\mathrm{a}\end{array}$ & & $\begin{array}{c}0.35 \\
\mathrm{a}\end{array}$ & $\begin{array}{c}0.36 \\
a\end{array}$ & $\begin{array}{c}0.35 \\
a\end{array}$ & \\
\hline Mean (water) & & & $0.38 \mathrm{a}$ & & & & $0.35 \mathrm{~b}$ & \\
\hline
\end{tabular}

- Mean value for each species within each water level

i Mean value for cach shade level within each water level

Means with the same letter are not significantly different at: $=0.05$

\section{Leaf potassium content (LPC)}

Leaf potassium content showed highly significant $(p<0.001)$ variation between species, but was not significantly affected by either shade or water regimes. Alstonia showed a higher LPC under both water regimes while Acacia had lower LPC values (Table 7). Although the main effect of shade regimes on LPC was not significant at $p=0.05$, there was a significant shade $\mathrm{x}$ water regime interaction. LPC showed a decreasing trend with increasing shade under well-watered conditions while the opposite trend was observed under water stress (Table 7).

\section{Leaf water potential $(\Psi)$}

As the comparative variation of $\Psi$ on the three days of measurements was similar, only the $\Psi$ data on 04 September 1998 are presented here. The main effects of species and shade on $\Psi$ was highly significant $(p<0.001)$. Although the main effect 
Table 6: Leaf nitrogen content ( $\mathrm{mg} \mathrm{g}^{-1}$ leaf dry weight) of different tree species under varying shade and water regimes

\begin{tabular}{|c|c|c|c|c|c|c|c|c|}
\hline \multirow{2}{*}{ Tree species } & \multicolumn{4}{|c|}{ Well - watered } & \multicolumn{4}{|c|}{ Water - stressed } \\
\hline & Open & Med. & Higrh & Mean' & Open & Med. & High & Mean \\
\hline $\begin{array}{l}\text { Alstomia } \\
\text { macrophylla }\end{array}$ & 3.32 & 4.71 & 4.98 & $\begin{array}{c}4.66 \\
\mathrm{~A}\end{array}$ & 0.28 & 0.44 & 0.45 & $\begin{array}{r}5.02 \\
\mathrm{AB}\end{array}$ \\
\hline $\begin{array}{l}\text { Artocarpus } \\
\text { hecterophyllus }\end{array}$ & 2.96 & 4.58 & 4.86 & $\begin{array}{c}4.46 \\
\mathrm{~A}\end{array}$ & 0.35 & 0.38 & 0.34 & $\begin{array}{c}4.48 \\
B\end{array}$ \\
\hline $\begin{array}{l}\text { Acacia } \\
\text { auriculiformis }\end{array}$ & 1.94 & 1.80 & 3.16 & $\begin{array}{c}2.30 \\
\mathrm{~B}\end{array}$ & 0.28 & 0.32 & 0.27 & $\begin{array}{c}4.55 \\
B\end{array}$ \\
\hline $\begin{array}{l}\text { Azadiracta } \\
\text { indica }\end{array}$ & 6.04 & 4.20 & 5.42 & $\begin{array}{c}5.22 \\
\mathrm{~A}\end{array}$ & 0.28 & 0.32 & 0.28 & $\begin{array}{c}6.12 \\
\mathrm{~A}\end{array}$ \\
\hline $\begin{array}{l}\text { Terminalia } \\
\text { arjuma }\end{array}$ & 4.45 & 4.90 & 4.50 & $\begin{array}{c}4.61 \\
\mathrm{~A}\end{array}$ & 0.54 & 0.38 & 0.43 & $\begin{array}{r}5.00 \\
A B\end{array}$ \\
\hline $\begin{array}{l}\text { Mean } \\
\text { (shade) }\end{array}$ & $\begin{array}{c}3.74 \\
\text { a }\end{array}$ & $\begin{array}{c}4.03 \\
\mathrm{ab}\end{array}$ & $\begin{array}{c}4.98 \\
a \\
\end{array}$ & & $\begin{array}{c}5.46 \\
a \\
\end{array}$ & $\begin{array}{c}4.88 \\
\mathrm{a}\end{array}$ & $\begin{array}{c}4.78 \\
\mathrm{a} \\
\end{array}$ & \\
\hline Mean (water) & & & $4.26 \mathrm{a}$ & & & & $5.04 \mathrm{~b}$ & \\
\hline
\end{tabular}

- Mean value for each species within each water level

- Mean value for each shade level within each water: level

Meams with the satno letter are not significanlly different at $\mathrm{p}=0.05$

of water regimes was not significant at $p=0.05$, there were highly significant $(p<0.001)$ water $x$ species and water $x$ species $x$ shade interactions. Alstonia had significantly $(p<0.05)$ greater $\Psi$ than the rest of the tree species under both water regimes (Table 8). In contrast, Acacia and Azadiracta had lower $\Psi$ values under both water levels. In well-watered conditions, $\Psi$ showed an increasing trend with increasing shade with the highest being found at high shade (Table 8). A similar pattern was found under water stress as well. $\Psi$ of individual species showed different patterns of response to increasing shade and the response pattern varied with the water regime as well. For example, under well-watered conditions, Artocarpus and Acacia increased their $\Psi$ with increasing shade whereas Azadiracta showed the opposite trend (Table 8). In contrast, under water stress, while Artocarpus showed the same increasing trend of $\Psi$ with increasing shade, $\Psi$ of Acacia decreased with increasing shade. 
Table 7: Leaf potassium content ( $\mathrm{mg}^{-1}$ leaf dry weight) of different tree species under varying shade and water regimes

\begin{tabular}{|c|c|c|c|c|c|c|c|c|}
\hline \multirow{2}{*}{ Tree species } & \multicolumn{4}{|c|}{ Wel] - watered } & \multicolumn{4}{|c|}{ Water - stressed } \\
\hline & Open & Med. & High & Mean ${ }^{*}$ & Open & Med. & High & Mean' ${ }^{*}$ \\
\hline $\begin{array}{l}\text { Alstonia } \\
\text { macrophylla }\end{array}$ & 2.30 & 2.18 & 2.18 & $\begin{array}{c}2.22 \\
\mathrm{~A}\end{array}$ & 1.94 & 2.10 & 2.30 & $\begin{array}{l}2.1 .2 \\
\mathrm{AB}\end{array}$ \\
\hline $\begin{array}{l}\text { Artocarpus } \\
\text { heterophyllus }\end{array}$ & 1.98 & 2.16 & 2.08 & $\begin{array}{l}2.08 \\
\mathrm{BC}\end{array}$ & 1.04 & 2.20 & 2.10 & $\begin{array}{c}1.78 \\
\mathrm{C}\end{array}$ \\
\hline $\begin{array}{l}\text { Acacia } \\
\text { auriculiformis }\end{array}$ & 2.18 & 1.86 & 1.28 & $\begin{array}{c}1.78 \\
D\end{array}$ & 1.98 & 1.62 & 1.92 & $\begin{array}{l}1.84 \\
\mathrm{BC}\end{array}$ \\
\hline $\begin{array}{l}\text { Azadiracta } \\
\text { indica }\end{array}$ & 2.30 & 1.96 & 2.16 & $\begin{array}{l}2.14 \\
A B\end{array}$ & 2.20 & 2.12 & 1.96 & $\begin{array}{l}2.10 \\
A B\end{array}$ \\
\hline $\begin{array}{l}\text { Terminalia } \\
\text { arjunce }\end{array}$ & 1.98 & 1.80 & 2.14 & $\begin{array}{l}1.98 \\
\mathrm{C}\end{array}$ & 2.46 & 1.96 & 2.12 & $\begin{array}{c}2.18 \\
\mathrm{~A}\end{array}$ \\
\hline $\begin{array}{l}\text { Meant } \\
\text { (shade) } \\
\end{array}$ & $\begin{array}{c}2.14 \\
a \\
\end{array}$ & $\begin{array}{c}2.00 \\
b\end{array}$ & $\begin{array}{c}1.98 \\
\mathrm{~b}\end{array}$ & & $\begin{array}{c}1.92 \\
a\end{array}$ & $\begin{array}{c}2.00 \\
\mathrm{a}\end{array}$ & $\begin{array}{c}2.08 \\
a\end{array}$ & \\
\hline Mean (water) & & & $2.04 \mathrm{a}$ & & & & $2.00 \mathrm{a}$ & \\
\hline
\end{tabular}

Mean value for each species within each water level

Mean value fir each shade level within each water level.

Means with the same letter are not significantly different at $p=0.05$

\section{Leaf stomatal conductance $\left(\mathrm{g}_{1}\right)$}

Because of the observed similarity of $g_{1}$ data on the three days of measurement, only the data on 03 September 1998 are shown in Table 9. Main effects of all the factors tested (i.e. species, shade and water regimes) on $g_{1}$ were highly significant $(p<0.0001)$. Under well-watered conditions, the highest $\mathrm{g}_{1}$ was shown by Alstonia whereas Acacia showed the highest $g_{1}$ under water-stressed conditions (Table 9). On the other hand, Azadiracta had the lowest g, under both water regimes. There was a significant decrease of $g_{1}$ with increasing shade under both well-watered and water-stressed conditions. As compared to well-watered conditions, water stress caused a significant decrease of $g_{1}$ in all species under all shade regimes (Table 9). The proportional decreases of $\mathrm{g}$, due to water stress was greater under open conditions as compared to medium-and high shade. Under water-stressed conditions, $g_{1}$ of all species showed a decreasing trend with increasing shade. However, under well-watered conditions, only Acacia showed a similar trend 
(Table 9). Azadiracta and Terminalia showed a slightincrease in g; under medium shade (as compared to open conditions) whereas Alstonia and Artocarpus showed increases when shade was increased from medium to high shade.

Table 8: Leaf water potential (bars) of different twee species under varying shade and water regimes

\begin{tabular}{|c|c|c|c|c|c|c|c|c|}
\hline \multirow{2}{*}{ Tree species } & \multicolumn{4}{|c|}{ Well - watered } & \multicolumn{4}{|c|}{ Water - stressed } \\
\hline & Open & Med. & High & Mean & Open & Med. & High & Mean \\
\hline $\begin{array}{l}\text { Alstoniza } \\
\text { macrophylla }\end{array}$ & -0.90 & -1.65 & -1.05 & $\begin{array}{c}-1.20 \\
\mathrm{~A}\end{array}$ & -4.23 & -3.15 & -2.90 & $\begin{array}{c}-3.43 \\
\mathrm{~A}\end{array}$ \\
\hline $\begin{array}{l}\text { Artocarpuss } \\
\text { heterophyllus.s }\end{array}$ & -16.0 & -10.0 & -3.95 & $\begin{array}{c}-9.98 \\
\mathrm{C}\end{array}$ & -8.35 & -4.70 & -6.48 & $\begin{array}{c}-6.51 \\
B\end{array}$ \\
\hline $\begin{array}{l}\text { Acacia } \\
\text { auriculiformis }\end{array}$ & -12.4 & -10.2 & -7.95 & $\begin{array}{c}-10.2 \\
C\end{array}$ & -9.68 & -11.0 & -10.4 & $\begin{array}{c}-10.3 \\
D\end{array}$ \\
\hline $\begin{array}{l}\text { Azadiracta } \\
\text { indica }\end{array}$ & -8.85 & -8.13 & -11.7 & $\begin{array}{c}-9.57 \\
\mathrm{C}\end{array}$ & -12.0 & -11.5 & -9.75 & $\begin{array}{c}-11.1 \\
D\end{array}$ \\
\hline $\begin{array}{l}\text { Teminalia } \\
\text { arjuna }\end{array}$ & -4.05 & -9.25 & -6.65 & $\begin{array}{c}-6.65 \\
B\end{array}$ & -7.70 & -9.20 & -8.45 & $\begin{array}{c}-8.45 \\
C\end{array}$ \\
\hline $\begin{array}{l}\text { Mean } \\
\text { (shade) }\end{array}$ & $\begin{array}{c}-8.44 \\
b\end{array}$ & $\begin{array}{c}-7.85 \\
b\end{array}$ & $\begin{array}{c}-6.25 \\
\mathrm{a}\end{array}$ & & $\begin{array}{c}-9.29 \\
a\end{array}$ & $\begin{array}{c}-7.89 \\
a\end{array}$ & $\begin{array}{c}-7.60 \\
a\end{array}$ & \\
\hline Mean (water) & & & -7.52 & & & -7.96 & & \\
\hline
\end{tabular}

Mean value fir each species within each water level

t Mean value for each shade level within each water level

Means with the samse letter are not significantly different at $p=0.05$

\section{Correlations between biomass gain and leaf characters}

Table 10 shows the linear correlation coefficients between biomass gain (absolute and relative) and the measured leaf characters. Leaf water potential, stomatal conductance and chlorophyll content showed significant $(p<0.05)$ correlations with both absolute biomass gain and RGR. On the other hand, there were no significant correlations between absolute or relative biomass gain and leaf nutrient contents. 
Table 9: Leaf stomatal conductance $\left(\mathrm{mmol} \mathrm{m} \mathrm{m}^{-2} \mathrm{~s}^{-1}\right)$ of different tree species under varying shade and water regimes

\begin{tabular}{|c|c|c|c|c|c|c|c|c|}
\hline \multirow{2}{*}{ Tree species } & \multicolumn{4}{|c|}{ Well - watered } & \multicolumn{3}{|c|}{ Water - stressed } & \multirow[b]{2}{*}{ Mean $^{+}$} \\
\hline & Open & Med. & High & Mean & Open & Med. & High & \\
\hline $\begin{array}{l}\text { Alstonia } \\
\text { macrophylla }\end{array}$ & 260.1 & 82.05 & 61.66 & $\begin{array}{c}134.6 \\
\mathrm{~A}\end{array}$ & 77.65 & 53.50 & 30.0 & $\begin{array}{c}53.72 \\
B\end{array}$ \\
\hline $\begin{array}{l}\text { Artocarpus } \\
\text { heterophyllus }\end{array}$ & 235.5 & 46.50 & 66.00 & $\begin{array}{l}116.2 \\
A B\end{array}$ & 62.75 & 54.95 & 25.6 & $\begin{array}{c}4.7 .77 \\
\mathrm{C}\end{array}$ \\
\hline $\begin{array}{l}\text { Acacia } \\
\text { auriculiformis }\end{array}$ & 167.0 & 70.15 & 54.95 & $\begin{array}{c}97.37 \\
B C\end{array}$ & 82.50 & 54.60 & 64.95 & $\begin{array}{c}67.35 \\
\mathrm{~A}\end{array}$ \\
\hline $\begin{array}{l}\text { Azadiracta } \\
\text { indica }\end{array}$ & 58.60 & 77.20 & 46.90 & $\begin{array}{c}60.73 \\
\mathrm{D}\end{array}$ & 61.35 & 31.38 & 27.50 & $\begin{array}{c}40.48 \\
\mathrm{C}\end{array}$ \\
\hline $\begin{array}{l}\text { Terminaliar } \\
\text { arjuna }\end{array}$ & 78.15 & 84.30 & 72.50 & $\begin{array}{c}78.32 \\
\mathrm{CD}\end{array}$ & 65.05 & 63.50 & 56.90 & $\begin{array}{c}61.82 \\
\text { B }\end{array}$ \\
\hline $\begin{array}{l}\text { Mean: } \\
\text { (shade) }\end{array}$ & $\begin{array}{c}160.0 \\
a\end{array}$ & $\begin{array}{c}72.04 \\
b\end{array}$ & $\begin{array}{c}60.30 \\
b\end{array}$ & & $\begin{array}{c}69.86 \\
\mathrm{a}\end{array}$ & $\begin{array}{c}51.59 \\
b\end{array}$ & $\begin{array}{c}40.99 \\
\mathrm{c}\end{array}$ & \\
\hline Mean (Water) & & & $97.43 \mathrm{a}$ & & & & $54.15 \mathrm{~b}$ & \\
\hline
\end{tabular}

Mean value tio each species within each water ]evel

Mean value for each shade level within each water level

Means with the same letiter are not significantly different at $p=0.05$

\section{Contributions of leaf characters to variation in biomass gain}

Table 11 shows the estimated significant contributions of leaf characters to the oloserved variation of biomass gain. For both absolute and relative biomass gain, the highest contributions were made by leaf water potential (which accounted for: $34 \%$ and $27 \%$ of the variation) and stomatal conductance (18\% and $28 \%$ ).

\section{DISCUSSION}

Findings of the present study provide important indications about the physiological basis of variation in growth potential of early-and late-successional tree species. Measurements of biomass gain showed that inter-species variation was a stronger determinant of biomass production, in both absolute and relative terms, than the effects of either shade or water. This is because the comparative performance of different tree species did not differ much between open, medium and high shade 
Table 10: Linear correlation coefficients between biomass gain and leaf characters in different tree species under varying shade and water regimes

\begin{tabular}{lcccc}
\hline $\begin{array}{l}\text { Leaf } \\
\text { character }\end{array}$ & $\begin{array}{c}\text { Total biomass } \\
\text { gain }\end{array}$ & Probability $^{*}$ & $\begin{array}{c}\text { Relative } \\
\text { growth rate }\end{array}$ & Probability" \\
\hline $\begin{array}{l}\text { Chlorophy]1 } \\
\text { content }\end{array}$ & 0.532 & 0.0025 & 0.375 & 0.0412 \\
$\begin{array}{l}\text { Water } \\
\text { potential }\end{array}$ & 0.581 & 0.0008 & 0.518 & 0.0033 \\
$\begin{array}{l}\text { Stomatal } \\
\text { conductance }\end{array}$ & 0.388 & 0.034 & 0.494 & 0.005 \\
$\begin{array}{l}\text { Nitrogen } \\
\text { content }\end{array}$ & 0.068 & 0.72 & -0.08 & 0.6729 \\
$\begin{array}{l}\text { Potassium } \\
\text { content }\end{array}$ & 0.220 & 0.242 & 0.055 & 0.7742 \\
\hline
\end{tabular}

Prohability of obtaining a correlation coefficient as large as or larger than that of given here hy chance aleme

Table 11: Factors contributing to the observed variation of biomass gain in different tree species under varying shade and water regimes

\begin{tabular}{lcccccr}
\hline & \multicolumn{2}{c}{$\begin{array}{c}\text { Absolute biomass gain (W) } \\
\text { Parameter } \\
\text { estimate }\end{array}$} & $\begin{array}{c}\text { Partial } \\
\mathrm{R}^{2}\end{array}$ & Prob. & \multicolumn{3}{c}{ Relative growth rate (RGR) } \\
& Parameter & Partial & Prob. \\
estimate & $\mathrm{R}^{2}$ & \\
\hline $\begin{array}{l}\text { Leaf water } \\
\text { potential }\end{array}$ & 0.827 & 0.338 & 0.0008 & 0.412 & 0.269 & 0.0033 \\
$\begin{array}{l}\text { Stomatal } \\
\text { conductance }\end{array}$ & 0.049 & 0.1808 & 0.0036 & 0.027 & 0.278 & 0.0004 \\
\hline
\end{tabular}

Probahility of ohtaining a regression parameter estimate as large as or larger than that of given here by thance alone.

Note: Only these parameter estimales with a probahility value of less than $\left(0.150()\right.$ are given. $R^{2}$ values of the full regressions models were 0.557 (for W) and 0.563 (for RGR).

conditions or between well-watered and water-stressed conditions. For example, the pioneer species, Alstonia macrophylla showed the highest biomass gain under all levels of shade and water. However, the reduction of biomass gain (both absolute and relative) with shading (i.e. comparison between open and medium shade) was greater in eariy-successional species (eg. Alstonia) as compared to late-successional 
species (eg. Artocarpus), especially when water was not limiting. Despite a few exceptions, reductions in biomass gain due to water stress tended to be lower under medium and high shade as compared to open conditions. However, no species (including the late-successional species tested) showed greater biomass production potential under shaded conditions used in the present study. Likewise, in the shade levels maintained in the present experiment, the pioneers would still maintain the competitive advantage of their greater biomass production potential over the climax species. Therefore, the inability of the pioneers to regenerate under shaded conditions may not be due to a lower carbon balance under shade. Rather, it may be due to other factors such as inability to germinate under low light intensities with a higher far-red/red ratio as found in a forest understorey. ${ }^{4}$

The decrease of biomass gain with increasing shade was probably because of the lower total photosynthesis resulting from reduced radiant energy receipt. This observation agrees with a wide body of available literature. ${ }^{20-2:}$ The positive response shown by the biomass gain of both early-and late-successional species to increasing light shows that even the seedlings of climax species growing on the forest floor are able to increase their photosynthesis when occasional sunflecks occur. This agrees with the findings of Chazdon" and Pearcy et al. "that 30-60\% of carbon gain of understorey seedlings may occur during the short periods of sunflecks. Measurements of several leaf characters (j.e. chlorophyll and nutrient contents, water potential and stomatal conductance) enabled elucidation of the physiological basis of the observed variation in biomass gain in different tree species. Both correlation and multiple regression analysis showed that leaf water potentia] and stomatal conductance exerted a dominant influence on biomass gain of different tree species. For example, Alstonia, which had higher biomass gains, had higher $\Psi$ and $g_{1}$. In contrast, $\Psi$ and $g_{1}$ were lower in Azi diracta which showed lower biomass gains. Greater $\Psi$ permits a higher turgor within cells which enable al] physiological processes, ${ }^{24}$ including photosynthesis, to perform at higher rates. ${ }^{9.25}$ This was probably the reason for the observed positive relationship between biomass gain and $\Psi$. Moreover, greater $\Psi$ also increases $g_{1}^{26}$ which allows greater CO. uptake in to the leaves." This increased gas exchange enhances photosynthesis and biomass gain. In addition to the higher levels of $\Psi$ and $g_{1}$, Alstonia also had higher leaf chlorophyll and potassium contents which also could contribute towards its greater biomass production potential. Greater chlorophyll contents enhances light capture ${ }^{2 \pi}$ and when combined with greater $g_{1}$ and $\Psi$ allows greater photosynthesis. As potassium is required mainly for cellular transfer processes ${ }^{2 / 5}$ and maintaining favourab]e water relations, ${ }^{2 !}$ a higher leaf potassium content probably contributed towards the greater growth rates of Alstonia.

Leaf nitrogen content is an indication of the amount of photosynthetic enzymes present." Specifically, around 50\% of soluble protein in leaves consists of the primary carboxylating enzyme ribulose 1,5 bisphosphate carboxylase-oxygenase. ${ }^{31}$ Therefore, leaf njtrogen content is positively correlated with the maximum 
light-saturated photosynthetic rate. ${ }^{32 \% 4}$ However, except for species which form the upper canopy, light is very often a limiting factor in forests. Hence, having a high photosynthetic capacity through a greater leaf nitrogen content, would not achieve a greater biomass gain unless factors which enhance light capture (i.e. chlorophyll content) are also present at favourable levels. This was probably the reason for the absence of any significant correlation between biomass production potential and leaf nitrogen content in the species examined in the present experiment. A specific example was Azadiracta indica which had the highest leaf nitrogen content but had lower leaf chlorophyll contents and consequently lower biomass gains. However, having a greater light-saturated photosynthetic capacity through a higher leaf nitrogen content would enable species to maximize photosynthetic rates during sunflecks which provide high light intensities over short durations.

The observed reduction of LWR and increase of RWR in response to shade show that, in the species examined in the study, leaf growth was more sensitive to shading as compared to overall growth. Conversely, the sensitivity of root growth to increasing shade was less than that of overall growth.

Based on the results of the present study, it is concluded that early-successional species have a greater biomass production potential once they are established under any shade level above $30 \%$ of incident radiation. The greater biomass production potential was mainly due to higher leaf water potential and stomatal conductance. Therefore, pioneers may be used in the reforestation programmes not only under open conditions and large forest gaps, but also under shaded conditions. Pioneers such as Alstonia could be used to close the canopy gaps within a shorter time period. This is especially important in the wet zone forests of Sri Lanka as the high rainfall in this zone could cause significant soil erosion in forest gaps.

In contrast, for reforestation in the dry zone, slower-growing latesuccessional species such as Azadiracta and Artocarpus can be recommended for both larger and smaller forest gaps. As shown by this study, these slow-growing species respond positively to the higher levels of irradiance that penetrate on to the floor of dry zone forests. Recommendation of fast-growing pioneers such as Alstonia for reforestation in the dry zone should be done only with extreme caution. As biomass accumulation is directly proportional to water use through evapotranspiration, $\$$ fast-growing tree species could quickly deplete the limited soil moisture reservoir in the dry zone.

\section{Acknowledgement}

The assistance provided by A.G. Chandrapala and K.B. Attanayake is acknowledged. 


\section{References}

1. Legg C. \& Jewell N. (1995). A 1:50,000-scale forest map of Sri Lanka: the basis for a national forest geographic information system. The Sri Lanka Forester, Special Issue - Remote Sensing, pp: 3-24.

2. Tilman D. (1986). Evolution and differentiation in terrestrial plant communities: the importance of the soil resource: light gradient. In: Community Ecology. (Eds. J. Diamond \& T.J. Case). pp. 359-380. Harper and Row, New York.

3. Perry D.A. (1994). Forest Ecosystems. The John Hopkins University Press, London. 649 pp.

4. Bazzaz F.A. (1991). Regeneration of tropical forests: physiological responses of pioneer and secondary species. In: Rain Forest Regeneration and. Management. (Eds. A. Gomez-Pompa, T.C. Whitmore \& M. Hadley). pp). 91-118. UNESCO, Paris.

5. Chazdon R.L. (1988). Sunflecks and their importance to forest understorey plants. Advances in Ecological Research 18: 1-63.

6. Pearcy R.W., Chazdon R.L., Gross L.J. \& Mott K.A. (1994). Photosynthetic utilization of sunflecks: a temporally patchy resource on a time scale of seconds to minutes. In: Exploitation of Environmental Heterogeneity by Plants: Ecophysiological Processes Above-and Belowground. (Eds. M.M. Caldwell \& R.W. Pearcy). pp. 175-208. Academic Press, California, USA.

7. Jones H.G. (1992). Plants and Microclimate: A quantitative approach to environmental plant physiology. $2^{\text {urd }}$ Edition. Cambridge University Press, UK. $428 \mathrm{pp}$.

8. Farquhar G.D. \& Sharkey T.D. (1982). Stomatal conductance and photosynthesis. Annual Review of Plant Physiology' 33: 317-345.

9. Lawlor D.W. (1995). The effects of water deficit on photosynthesis. In: Environment and Plant Metabolism: Flexibility and Acclimation. (Ed. N. Smirnoff). pp. 129-160. Bios Scientific Publishers, UK.

10. Turner N.C. (1986). Crop water deficits: a decade of progress. Advances in Agronomy 39: 1-51.

11. Panabokke C.R. (1996). Soils and Agro-ecological Environments of Sri Lanka. Natural Resources, Energy and Science Authority of Sri Lanka. 220 pp. 
12. Little T.M. \& Hills F.J. (1978). Agricultural Experimentation: Design and Analysis. John Wiley and Sons, New York. $350 \mathrm{pr}$.

13. Scholander P.F., Hammel H.T., Bradstreet E.D. \& Hemmingsen E.A. (1965). Sap pressure in vascular plants. Negative hydrostatic pressure can be measured in plants. Science 148: 339-346.

14. Turner N.C. (1981). Techniques and experimental approaches for the measurement of plant water status. Plant and Soil 58: 341-366.

1.5. Moore T.C. (1981). Research Experiences in Plant Physiology: A Laboratory Manuckl. 2ul Edition. Springer-Verlag, New York. 348 pp.

16. Hunt R. (1978). Plank Grouth. Analysis. Studies in Biology no. 96. Edward Arrold, London. $67 \mathrm{pp}$.

17. Hesse P.R. (1971). A textbook of soil chemical analysis. John Murray, London.

18. Davidescu D. \& Davidescu V. (1982). Evaluation of fertility by plant and soil ancrlysis. Abacus Press, U.K. 560 pp.

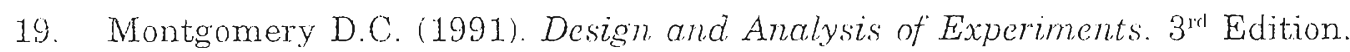
John Wiley \& Sons, New York. 649 pp.

20. Boardman N.K. (1977). Comparative photosynthesis of sun and shade plants. Annual Review of Plant Physiology 28: 355-377.

21. Bjorkman O. (1981). Responses to different quantum flux densities. In: Physiological Plant Ecology. I. Responses to the Physical Environment. Encyclopaedia of Plant Physiology, New Series, Vol. 12A. (Eds. O.I. Lange, P.S. Nobel, C.B. Osmond \& H. Ziegler). pp. 57-107. Springer-Verlag, New York.

22. Givnish T.J. (1988). Adaptation to sun and shade: A whole plant perspective. Australiani eournal of Plant Physiology 15: 63-92.

23. Pearcy R.W. \& Sims D.A. (1994). Photosynthetic acclimation to changing light environments: Scaling from leaf to whole plant. In: Exploitation of Environmental Heterogeneity by Plants: Ecophysiological Processes Above-and Belowground. (Eds. M.M. Caldwell \& R.W. Pearcy). pp. 145-174. Academic Press, California, USA.

24. Sutcliffe J.F. (1986). Water relations of plant cells. In: Plant Physiology: A Treatise. (Eds. F.C. Steward, J.F. Sutcliffe \& J.E. Dale). pp. 1-48. Academic Press, New York. 
25. Law or D.W. \& Uprety D.C. (1993). Effects of water stress on photosynthesis of crops and the biochemical mechanism. In: Photosynthesis: Photoreactions to Plant Productivity. (Eds. Y.P. Abrol, P. Mohanty \& Govindjee). pp. 419-449. Oxford and IBH Publishers, New Delhi.

26. Mansfield T.A. (1986). The physiology of stomata: New insights into old problems. In: Plant Physiology: A Treatise. (Eds. F.C. Steward, J.F. Sutcliffe \& J.E. Dale). pp. 155-224. Academic Press, New York.

27. Lawlor D.W. (1987). Photosynthesis: Metabolism, Control and Physiology. Longman, UTK. 262 pp.

28. Marschner H. (1995). Mineral Nutrition of Higher Plants. 2nd Edition. Academic Press, London. 889 pp.

29. Leigh R.A. \& Wyn Jones R.G. (1984). A hypothesis relating critical potassium concentrations for growth to the distribution and functions of this ion in the plant cell. Netu Phytologist 97: 1-13.

30. Sinclair T.R. (1991). Canopy carbon assimilation and crop radiation use efficiency dependence on leaf nitrogen content. In: Modelling Crop Photosynthesis - from Biochemistry to Canopy. (Eds. K.J. Boote \& R.S. Loomis). pp. 95-107. Crop Science Society of America, Madison, Wisconsin, USA.

31. Schmitt M.R. \& Edwards G.E. (1981). Photosynthetic capacity and nitrogen use efficiency of maize, wheat and rice: A comparison between $C_{3}$ and $\mathrm{C}_{1}$ photosynthesis. Journal of Experimental Botany 32: 459-466.

32. Sinclair T.R. \& Horie T. (1989). Leaf nitrogen, photosynthesis and crop radiation use efficiency. Crop Science 29: 90-98.

33. Field C. \& Mooney H.A. (1986). The photosynthesis-nitrogen relationship in wild plants. In: On the Economy of Form and Function. (Ed. T.J. Givnish). pp. 25-55. Cambridge University Press, Cambridge, U.K..

34. Evans J.R. (1989). Photosynthesis and nitrogen relationships in leaves of C3 plants. Oecologia 78: 9-19.

35. Calder I.R. (1996). Water use by forests at the plot and catchment levels. Commonwealth. Forestry Review 75: 19-30. 